\title{
THE MOTH FAUNA (LEPIDOPTERA: HETEROCERA) OF MEDVEDNICA NATURE PARK, CROATIA
}

\section{Toni Koren}

Hyla Association, Lipovac I 7, HR-10000 Zagreb, Croatia (e-mail: toni.koren@hhdhyla.hr)

Koren, T.: The moth fauna (Lepidoptera: Heterocera) of Medvednica Nature Park, Croatia. Nat. Croat., Vol. 29, No. 1, 37-54, Zagreb, 2020.

The paper gives the first overview of the moth fauna of Medvednica Nature Park, one that is based on the literature data and recent surveys carried out in the period from 2015 to 2019. The literature overview confirms the presence of 69 moth species recorded within the borders of the Nature Park. The survey recorded a total of 437 moth species in the area, 388 of them for the first time. In all, 455 species are now confirmed for Medvednica Nature Park. One Tortricidae species, Phtheochroa annae, is new for the fauna of Croatia. The area of Medvednica Nature Park can be now considered as one of the best explored Nature parks in Croatia, with the first checklist of its moth fauna. In most of the visited sites across Medvednica, habitat conditions are still favorable for the short-term survival of most species, but the overgrowing of meadows and forest edges, as well as intensive mowing of meadows, may dramatically worsen this situation during the next decades. Further moth surveys in this protected area would therefore be advisable.

Key words: Zagreb, diversity, distribution, conservation, protected area

Koren, T.: Fauna noćnih leptira (Lepidoptera: Heterocera) Parka prirode Medvednica. Nat. Croat., Vol. 29, No. 1, 37-54, Zagreb, 2020.

Prilog prikazuje prve rezultate istraživanja noćnih leptira Parka prirode Medvednica, temeljene na literaturnim podacima i recentnim istraživanjima provedenim između 2015. i 2019. godine. Pregledom literature potvrđena je prisutnost 69 vrsta zabilježenih unutar granica Parka prirode. U našim istraživanjima zabilježili smo 437 vrsta noćnih leptira, od kojih 388 prvi put na tom području. Ukupan broj vrsta noćnih leptira Parka prirode Medvednica sada sadrži 455 vrsta. Jedna vrsta iz porodice Tortricidae, Phtheochroa annae, nova je za faunu Hrvatske. Nakon ovog istraživanja noćnih leptira područje Parka prirode Medvednica može se smatrati jednim od najbolje istraženih zaštićenih područja u Hrvatskoj. Na većini posjećenih područja diljem Medvednice stanišni uvjeti povoljni su za kratkoročni opstanak većine vrsta, ali zarastanje livada i rubova šume, kao i intenzivna košnja vršnih livada, mogli bi u idućim desetljećima dramatično pogoršati ovu situaciju. Radi toga preporučujemo nastavak istraživanja noćnih leptira ovog vrijednog područja i u budućnosti.

Ključne riječi: Zagreb, raznolikost, rasprostranjenost, zaštita, zaštićeno područje

\section{INTRODUCTION}

Moths are one of the most prevalent terrestrial insects. They are also one of the most numerous groups of insects with more than 160,000 described species, but the total number of extant species is estimated to be around half a million (KRISTENSEN et al., 2006). Moths perform essential ecosystem services such as pollination, decomposition, and nutrient cycling and provide prey for birds and other vertebrates (SCHMIDT \& Ro- 
LAND, 2009; HAHN \& BRÜHL, 2016). In this respect, the knowledge about moth diversity and abundance is especially beneficial for protected or Natura 2000 areas.

In Croatia, protected areas of different kinds cover $7.95 \%$ of the territory. Among them, there are 11 Nature Parks. Of these, published results about the moth fauna exist only for Kopački Rit Nature Park where 201 species of mostly Macrolepidoptera have been recorded (Vignjević et al., 2010). For other nature parks, only historical records exist, but without any checklists or overviews.

Medvednica Nature Park, in the vicinity of Zagreb has never been in the focus of systematic moth surveys. The first records of the moths of Medvednica are found in the work of Ljudevit Vukotinović, in which he presents the records of 42 species for Mt. Medvednica (gora Zagrebačka) (Vuкотіnović, 1879). Although valuable, his work today must be considered with caution, with the knowledge of Lepidopteran literature from that time. Just as with the list of butterflies he provided; his list of moths contains a large number of species that are certainly not present in the area of Medvednica. For example, the species Scopula ternata (Acidalia fumata) has not yet been recorded in the fauna of Croatia, and the species Autophila cataphanes and Apopestes spectrum are now present only in the Mediterranean coastal area of Croatia, far away from Medvednica Nature Park. For this reason, we did not take his work into account when compiling the list of moths of Medvednica Nature Park.

Further records from Medvednica are given by GRUND (1916), who mentions only five species collected within the boundaries of the Nature Park. The most significant contribution to the moths of Medvednica was published by MLadinov (1958) in her review of the moth fauna of Zagreb, with 91 records of 54 different moth species that fall within the boundaries of Medvednica Nature Park. In a recent review of the collection of Vanda Kochansky - Devidé, there are four species of moths collected in the Medvednica area (Koren et al., 2018). In one of the papers of Lorkovic (1977) dedicated to moths, he mentions the species Xanthorhoe biriviata from the Pustodol area of Sljeme. Mladinov (1976) reports the findings of Tetheella fluctuosa and Thyris fenestrella from Mt. Medvednica. In an overview of the Noctuoidea species stored in the Lepidoptera collection of the Faculty of Forestry, KučInIć \& Hrašovec (1999) report nine species collected on Mt. Medvednica. After that, only a single species is reported from the area, MatošEvić et al. (2009) mention the findings of Argyresthia fundella.

In this contribution we provide the results of a more systematic survey of the moth fauna of Medvednica Nature Park, with the complete data from the literature. Some guidelines for the long-term protection of the moth fauna are also discussed.

\section{MATERIALS AND METHODS}

\section{Study area}

The Medvednica mountain range is located on the northern edge of Zagreb, the capital of Croatia. It is approximately 42 kilometers long, extending in a southwest-northeast direction. Because Medvednica is located on the very edge of the capital, the anthropogenic influence on almost the entire area is very significant and visible. Medvednica is a favorite destination for hikers and nature lovers. This is especially reflected in the large number of roads, forest roads, and also lawns and meadows created by deforestation and subsequent mowing. Today, almost all of the mountain range belon- 
gs to the Medvednica Nature Park (Medvednica NP), a protected area of 17,938 ha. The highest peak of the massif is Sljeme, 1,035 $\mathrm{m}$ a.s.l. The dominant habitat types within the park are forest, which covers $81 \%$ of the area, and the characteristics of forest communities vary with altitude and terrain exposure. In the lowlands, forests of oak and common hornbeam (Epimedio-Carpinetum betuli) are dominant. Most of the territory of Medvednica is covered with beech forests or mixed beech - fir forests. Less common are grassland (meadows, pastures), clearings, vineyards and orchards. In the area of the Medvednica Nature Park, at least 1205 plant taxa have been recorded (Dobrović et al., 2006). Unlike the flora, the fauna of Medvednica is largely unexplored, especially the invertebrate fauna.

\section{Moth survey}

Day flying moths were observed or caught with an entomological net during the day. Nightly active moths were attracted using standard Lepidoptera equipment. On most localities, up to six UV light tents were used. On average, four hours were spent on each locality on each date, depending on the season and climate conditions. In total, 16 localities were visited during this survey (Tab. 1, Fig. 1).

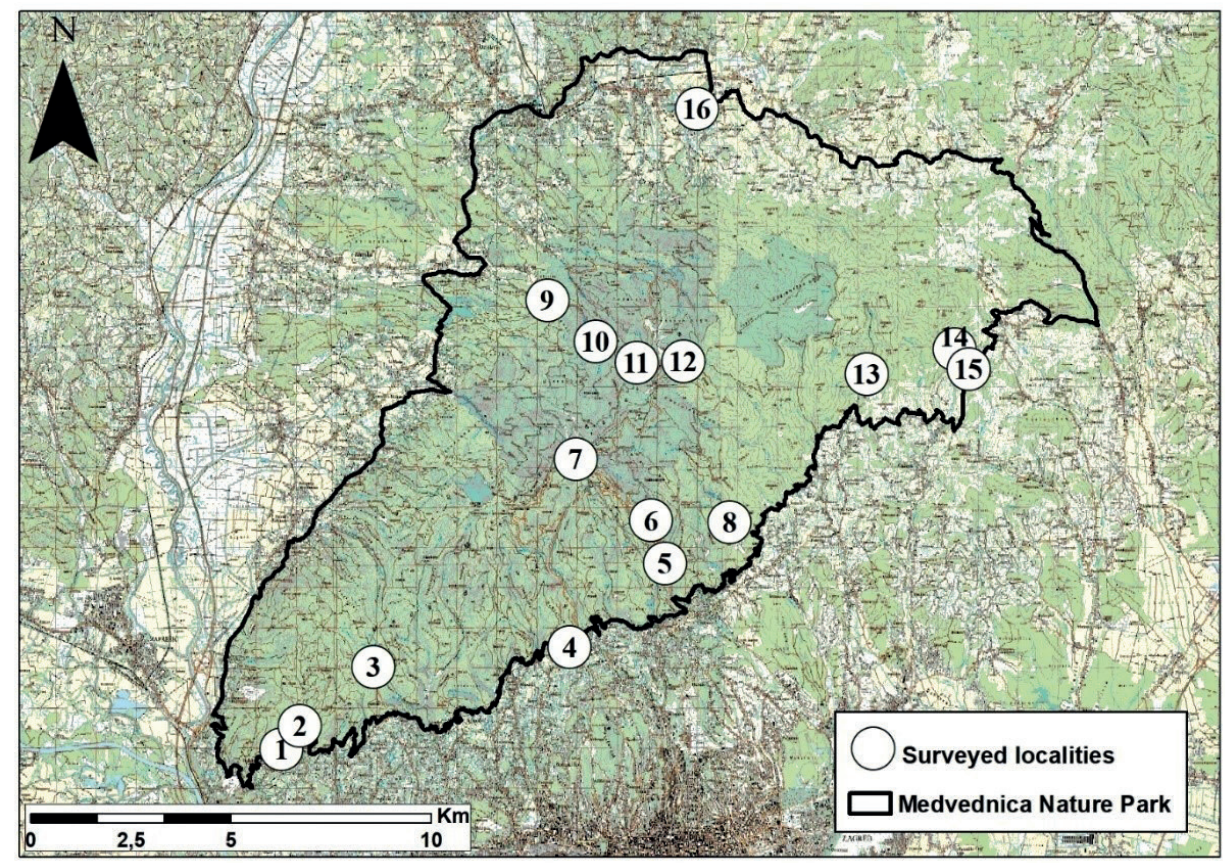

Fig. 1. Borders of Medvednica Nature park with surveyed localities. Numbers of localities correspond to the ones given in Tab. 1.

Where possible, species were identified in the field using standard identification keys. Only in cases where the identification was questionable were moths collected and identified in the lab using standard identification keys (e.g. Fibiger, 1997; HausmanN \& ViIDALEPP, 2012; MACEK et al., 2008, 2012; NowACKI, 1998; SlAMKA, 2006, 2008, 2013). Collected 
specimens are stored in the private Koren Collection in Zagreb. The correct identification of many species was done by dissection of internal genital structures. Isolated genitals were afterwards stored in micro vials in glycerin, on the same pin as specimen. The nomenclature follows the online database Fauna Europaea (DE Jong et al., 2014).

Tab. 1. Surveyed localities on Medvednica Nature Park.

\begin{tabular}{|c|c|c|c|c|c|}
\hline & Locality & Dates of visit & WGS84 N & WGS84 E & Altitude \\
\hline 1. & $\begin{array}{l}\text { Medvednica NP, Podsused, } \\
\text { forest, banks of the stream }\end{array}$ & 10.6.2019 & 45,833774 & 15,852638 & 353 \\
\hline 2. & $\begin{array}{l}\text { Medvednica NP, Bizek, over- } \\
\text { grown quarry }\end{array}$ & $\begin{array}{l}\text { 22.3.2016, 18.4.2019, } \\
\text { 3.9.2019 }\end{array}$ & 45,839028 & 15,858751 & 353 \\
\hline 3. & $\begin{array}{l}\text { Medvednica NP, Ponikve, moist } \\
\text { meadows and forest edge }\end{array}$ & 9.6.2019 & 45,852029 & 15,882501 & 371 \\
\hline 4. & $\begin{array}{l}\text { Medvednica NP, Medvedgrad, } \\
\text { surroundings of the city }\end{array}$ & 25.8.2019, 26.10.2019 & 45,856045 & 15,945654 & 717 \\
\hline 5. & $\begin{array}{l}\text { Medvednica NP, Adolfovac, near } \\
\text { Bliznec stream, forest edge }\end{array}$ & 5.9 .2019 & 45,874489 & 15,976490 & 256 \\
\hline 6. & $\begin{array}{l}\text { Medvednica NP, Adolfovac, } \\
\text { northern road, forest edge }\end{array}$ & 5.9.2019 & 45,884115 & 15,972212 & 660 \\
\hline 7. & $\begin{array}{l}\text { Medvednica NP, Tomislavov } \\
\text { dom, forest edge }\end{array}$ & 15.8.2019 & 45,898068 & 15,948272 & 717 \\
\hline 8. & $\begin{array}{l}\text { Medvednica NP, Markuševec, } \\
\text { overgrown quarry }\end{array}$ & 1.9.2019 & 45,883459 & 15,997450 & 660 \\
\hline 9. & $\begin{array}{l}\text { Medvednica NP, Kraljev vrh, } \\
\text { forest edge }\end{array}$ & $\begin{array}{l}\text { 14.6.2019, 10.8.2019, } \\
\text { 11.8.2019, 5.9.2019, } \\
4.10 .2019\end{array}$ & 45,933783 & 15,939735 & 308 \\
\hline 10. & $\begin{array}{l}\text { Medvednica NP, Kraljev Vrh, } \\
\text { overgrown quarry, forest edge }\end{array}$ & 11.4.2016 & 45,924341 & 15,955074 & 308 \\
\hline 11. & $\begin{array}{l}\text { Medvednica NP, Sljeme, Rezani } \\
\text { k., forest edge }\end{array}$ & 4.9 .2019 & 45,919504 & 15,968202 & 519 \\
\hline 12. & $\begin{array}{l}\text { Medvednica NP, Sljeme, } \\
\text { Krčevina, forest edge }\end{array}$ & 5.9.2019 & 45,919712 & 15,983323 & 519 \\
\hline 13. & $\begin{array}{l}\text { Medvednica NP, Jambrešić, } \\
\text { Vidovec, forest edge }\end{array}$ & 15.6.2019, 16.6.2019 & 45,916357 & 16,042220 & 307 \\
\hline 14. & $\begin{array}{l}\text { Medvednica, Vejalnica, forest } \\
\text { edge, dry meadows }\end{array}$ & $\begin{array}{l}\text { 13.4.2016, 16.5.2016, } \\
\text { 10.6.2016, 8.9.2016, } \\
4.10 .2017\end{array}$ & 45,921551 & 16,070460 & 458 \\
\hline 15. & $\begin{array}{l}\text { Medvednica NP, Vejalnica, } \\
\text { southern part, forest edge, dry } \\
\text { meadows }\end{array}$ & $\begin{array}{l}\text { 26.6.2015, 15.7.2019, } \\
27.8 .2019,21.10 .2019\end{array}$ & 45,917064 & 16,075008 & 458 \\
\hline 16. & $\begin{array}{l}\text { Medvednica NP, Stubički } \\
\text { Golubovec, Golubovečki Ribnjaci, } \\
\text { forest and wet meadows }\end{array}$ & 26.4.2016, 22.7.2019 & 45,976283 & 15,988628 & 218 \\
\hline
\end{tabular}

\section{RESULTS}

During this survey, we recorded a total of 437 species (Tab. 2), 388 of which are new to Medvednica Nature Park. The literature review revealed the presence of 69 species of moths in the Medvednica Nature Park area (Tab. 2). Together with the literature data, 455 moth species have been recorded in the area. 
The highest proportion of recorded species belongs to the typical forest edge species that can be recorded on such marginal forest as well as disturbed habitats in high numbers (e.g. Camptogramma bilineata, Campaea margaritaria). Also, generalists like Autographa gamma and Chiasmia clathrata were very common in most localities in the nature park. Wet grassland species are only marginally present in some small areas of Medvednica Nature Park (e.g. Idaea biselata, Deltote bankiana). Several dry grassland moths have also been found, and of those Gortyna flavago and Luperina dumerilii can be regarded as local in northern Croatia. While L. dumerilii is very common in coastal areas of the country it has only occasionally been recorded in northern Croatia (LoRKOvić, 1977).

One of the rarer Geometrid species encountered during this survey is Stegania dilectaria. This species is distributed in northern, middle and eastern Croatia (MiноcI, 2012). The last published specimens originating from Croatia were recorded around Varaždin in 1950 (Minoci \& Bregović, 2008).

The occurrence of Thera vetustata has only recently been confirmed in Croatia (MIHOCI, 2012). One specimen of the usually mountain species was recorded on Medvednica. However, the record is not unusual as the species is widely distributed in the montane parts of Croatia (author, pers. obs.).

One Tortricidae species, Phtheochroa annae Huemer, 1990, was recorded in Croatia for the first time during this survey (Fig. 2). So far, only a single female specimen has been recorded, on Vejalnica Meadows. The correct species identification was confirmed by the analysis of the genital structures (Fig. 3) (see HüEmer, 1990). This Tortricidae species has been described relatively recently when a revision of the species Phtheochroa rugosana (Hübner, 1799) was done, resulting in the description of five new species based on the difference in male and female genital structures (HüEmER, 1990). Phtheochroa annae inhabits Central and South-eastern Europe and has been recorded in $\mathrm{Au}-$ stria, Hungary, Romania and Greece (Hüemer, 1990).

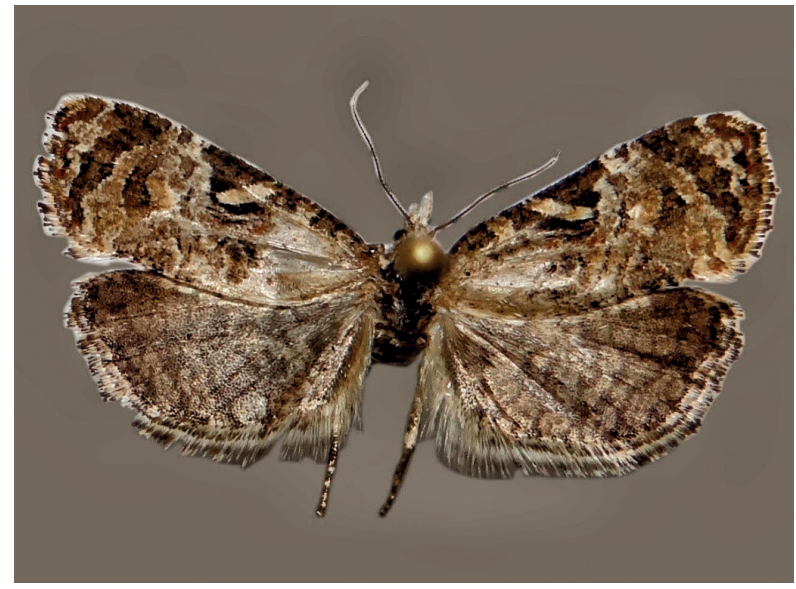

Fig. 2. Phtheochroa annae, from Vejalnica on Mt. Medvednica (Photo: T. Koren).

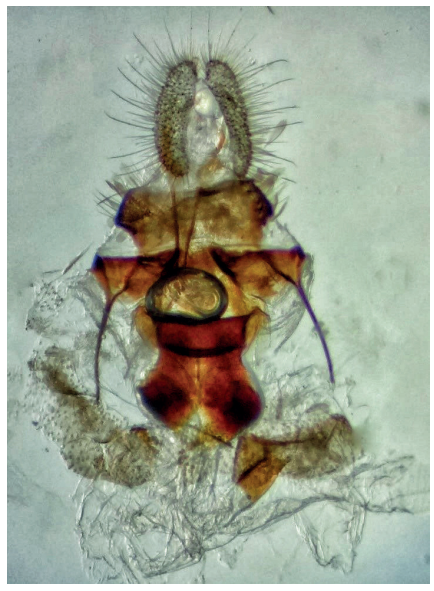

Fig. 3. Genitals of Phtheochroa annae, from Vejalnica on Mt. Medvednica (Photo: T. Koren). 
Tab. 2. List of moth species recorded during this survey. The locality numbers correspond to the ones given in Tab. 1. Species for which the identification is based on dissection of the genital structures are marked with an * symbol.

\begin{tabular}{|c|c|c|c|c|}
\hline & Family & Species & Locality & $\begin{array}{l}\text { Literature } \\
\text { records }\end{array}$ \\
\hline 1. & Argyresthiidae & Argyresthia fundella (Fischer von Röslerstamm, 1835) & & $\bullet$ \\
\hline 2. & Incurvariidae & Incurvaria masculella (Denis \& Schiffermüller, 1775) & 14 & \\
\hline 3. & Saturniidae & Aglia tau (Linnaeus, 1758) & $2,10,14$ & $\bullet$ \\
\hline 4. & & Antheraea yamamai (Guérin-Méneville, 1861) & $8,9,15$ & \\
\hline 5. & & Saturnia pavoniella (Scopoli, 1763) & 14 & \\
\hline 6. & Sphingidae & Agrius convolvuli (Linnaeus, 1758) & 8,15 & \\
\hline 7. & & Deilephila elpenor (Linnaeus, 1758) & 14 & \\
\hline 8. & & Deilephila porcellus (Linnaeus, 1758) & 14 & \\
\hline 9. & & Laothoe populi (Linnaeus, 1758) & 3 & \\
\hline 10. & & Macroglossum stellatarum (Linnaeus, 1758) & 7 & \\
\hline 11. & & Mimas tiliae (Linnaeus, 1758) & 9,13 & $\bullet$ \\
\hline 12. & & Smerinthus ocellata (Linnaeus, 1758) & 3 & \\
\hline 13. & & Sphinx ligustri Linnaeus, 1758 & 14,15 & \\
\hline 14. & & Sphinx pinastri Linnaeus, 1758 & 16 & \\
\hline 15. & Thyrididae & Thyris fenestrella (Scopoli, 1763) & & $\bullet$ \\
\hline 16. & Drepanidae & Cilixglaucata (Scopoli, 1763) & 14,15 & \\
\hline 17. & & Cymatophorina diluta (Denis \& Schiffermüller, 1775) & 14,15 & \\
\hline 18. & & Drepana curvatula (Borkhausen, 1790$)$ & $3,9,10$ & \\
\hline 19. & & Drepana falcataria (Linnaeus, 1758 ) & 3,9 & \\
\hline 20. & & Falcaria lacertinaria (Linnaeus, 1758) & 14 & \\
\hline 21. & & Habrosyne pyritoides (Hufnagel, 1766) & $1,9,13,15$ & \\
\hline 22. & & Polyploca ridens (Fabricius, 1787) & 2,14 & \\
\hline 23. & & Tethea or (Denis \& Schiffermüller, 1775) & $3,15,16$ & \\
\hline 24. & & Tetheella fluctuosa (Hübner, 1803) & & $\bullet$ \\
\hline 25. & & Thyatira batis (Linnaeus, 1758) & $1,9,13,14,16$ & \\
\hline 26. & & Watsonalla binaria (Hufnagel, 1767) & $8,9,14,15$ & \\
\hline 27. & & Watsonalla cultraria (Fabricius, 1775) & 14,15 & $\bullet$ \\
\hline 28. & Geometridae & Abraxas sylvata (Scopoli, 1763) & & $\bullet$ \\
\hline 29. & & Acasis viretata (Hübner, 1799) & $2,10,14,15,16$ & \\
\hline 30. & & Aethalura punctulata (Denis \& Schiffermüller, 1775) & 16 & \\
\hline 31. & & Alcis repandata (Linnaeus, 1758) & 13 & $\bullet$ \\
\hline 32. & & Alsophila aescularia (Denis \& Schiffermüller, 1775) & 10 & \\
\hline 33. & & Angerona prunaria (Linnaeus, 1758) & $\begin{array}{c}1,3,8,9,13,14,15 \\
16\end{array}$ & \\
\hline 34. & & Aplocera praeformata (Hübner, 1826) & 14 & \\
\hline 35. & & Artiora evonymaria (Denis \& Schiffermüller, 1775) & 2,8 & \\
\hline 36. & & Ascotis selenaria (Denis \& Schiffermüller, 1775) & $14,15,16$ & \\
\hline 37. & & Asthena albulata (Hufnagel, 1767) & 3 & \\
\hline 38. & & Asthena anseraria (Herrich-Schäffer, 1855) & 9,13 & \\
\hline 39. & & Biston betularia (Linnaeus, 1758) & 9,15 & \\
\hline 40. & & Cabera exanthemata (Scopoli, 1763) & $8,9,14,16$ & $\bullet$ \\
\hline 41. & & Cabera pusaria (Linnaeus, 1758) & 3,9 & $\bullet$ \\
\hline 42. & & Campaea margaritaria (Linnaeus, 1761) & $\begin{array}{c}1,2,3,5,6,8,9,11 \\
12,13,14,15\end{array}$ & $\bullet$ \\
\hline 43. & & Camptogramma bilineata (Linnaeus, 1758) & $2,8,9,13,14,15$ & \\
\hline 44. & & Cataclysme riguata (Hübner, 1813) & 14,15 & \\
\hline
\end{tabular}




\begin{tabular}{|c|c|c|c|c|}
\hline & Family & Species & Locality & $\begin{array}{l}\text { Literature } \\
\text { records }\end{array}$ \\
\hline 45. & & Catarhoe cuculata (Hufnagel, 1767) & $2,14,15,16$ & $\bullet$ \\
\hline 46. & & Catarhoe rubidata (Denis \& Schiffermüller, 1775) & 9 & \\
\hline 47. & & Cepphis advenaria (Hübner, 1790) & 13 & \\
\hline 48. & & Chiasmia clathrata (Linnaeus, 1758) & $3,7,14,15,16$ & $\bullet$ \\
\hline 49. & & Chlorissa cloraria (Hübner, 1813) & 14 & \\
\hline 50. & & Chloroclysta siterata (Hufnagel, 1767) & $10,14,15,16$ & \\
\hline 51. & & Chloroclystis v-ata (Haworth, 1809) & $14,15,16$ & \\
\hline 52. & & Cidaria fulvata (Forster, 1771) & 14 & $\bullet$ \\
\hline 53. & & Cleora cinctaria (Denis \& Schiffermüller, 1775) & 14 & \\
\hline 54. & & Colostygia olivata (Denis \& Schiffermüller, 1775) & $5,6,8$ & $\bullet$ \\
\hline 55. & & Colostygia pectinataria (Knoch, 1781) & $3,9,13,14,15$ & \\
\hline 56. & & Colotois pennaria (Linnaeus, 1761) & 15 & \\
\hline 57. & & Comibaena bajularia (Denis \& Schiffermüller, 1775) & 13 & \\
\hline 58. & & Cosmorhoe ocellata (Linnaeus, 1758) & $3,9,12,13,14,15$ & \\
\hline 59. & & Crocallis elinguaria (Linnaeus, 1758) & $5,6,9,14$ & \\
\hline 60. & & Cyclophora albiocellaria (Hübner, 1789) & 15 & \\
\hline 61. & & Cyclophora annularia (Fabricius, 1775) & $2,3,8,13,14,16$ & \\
\hline 62. & & Cyclophora linearia (Hübner, 1799) & $8,9,13,14$ & $\bullet$ \\
\hline 63. & & Cyclophora porata (Linnaeus, 1767) & 3 & \\
\hline 64. & & Cyclophora punctaria (Linnaeus, 1758) & $13,15,16$ & \\
\hline 65. & & Cyclophora ruficiliaria (Herrich-Schäffer, 1855) & 14 & \\
\hline 66. & & Earophila badiata (Denis \& Schiffermüller, 1775) & 10 & \\
\hline 67. & & Ecliptopera silaceata (Denis \& Schiffermüller, 1775) & 9 & $\bullet$ \\
\hline 68. & & Ectropis crepuscularia (Denis \& Schiffermüller, 1775) & $2,10,14,15$ & \\
\hline 69. & & Ematurga atomaria (Linnaeus, 1758) & $2,7,14,15$ & $\bullet$ \\
\hline 70. & & Ennomos quercinaria (Hufnagel, 1767) & & $\bullet$ \\
\hline 71. & & Ennomos fuscantaria (Haworth, 1809) & 8 & \\
\hline 72. & & Epione repandaria (Hufnagel, 1767) & 9 & \\
\hline 73. & & Epirrhoe alternata (Muller, 1764) & $3,9,14,15$ & \\
\hline 74. & & Epirrhoe tristata (Linnaeus, 1758) & & - \\
\hline 75. & & Epirrhoe galiata (Denis \& Schiffermüller, 1775) & $13,14,15$ & \\
\hline 76. & & Erannis defoliaria (Clerck, 1759) & 15 & \\
\hline 77. & & Euchoeca nebulata (Scopoli, 1763) & $1,3,9,13$ & \\
\hline 78. & & Eupithecia centaureata (Denis \& Schiffermüller, 1775) & 14,15 & \\
\hline 79. & & Eupithecia ericeata (Rambur, 1833) & 14 & \\
\hline 80. & & Eupithecia icterata (de Villers, 1789) & 5,8 & \\
\hline 81. & & Eupithecia insigniata (Hübner, 1790) & 14 & \\
\hline 82. & & Eupithecia linariata (Denis \& Schiffermüller, 1775) & 15 & \\
\hline 83. & & Fagivorina arenaria (Hufnagel, 1767) & 14,15 & $\bullet$ \\
\hline 84. & & Gandaritis pyraliata (Denis \& Schiffermüller, 1775) & 14 & \\
\hline 85. & & Gnophos furvata (Denis \& Schiffermüller, 1775) & 15 & $\bullet$ \\
\hline 86. & & Gymnoscelis rufifasciata (Haworth, 1809) & 9,14 & \\
\hline 87. & & Heliomata glarearia (Denis \& Schiffermüller, 1775) & 14,15 & \\
\hline 88. & & Hemistola chrysoprasaria (Esper, 1795) & 15 & \\
\hline 89. & & Holoterpna pruinosata (Staudinger, 1897) & 15 & \\
\hline 90. & & Horisme radicaria (de La Harpe, 1855) & 14 & \\
\hline 91. & & Horisme tersata (Denis \& Schiffermüller, 1775) & 15 & \\
\hline 92. & & Horisme vitalbata (Denis \& Schiffermüller, 1775) & 14 & \\
\hline 93. & & Hydrelia flammeolaria (Hufnagel, 1767) & 9,14 & \\
\hline
\end{tabular}




\begin{tabular}{|c|c|c|c|c|}
\hline & Family & Species & Locality & $\begin{array}{c}\text { Literature } \\
\text { records }\end{array}$ \\
\hline 94. & & Hydria cervinalis (Scopoli, 1763) & 14 & \\
\hline 95. & & Hydriomena furcata (Thunberg, 1784) & & $\bullet$ \\
\hline 96. & & Hydriomena impluviata (Denis \& Schiffermüller, 1775) & $3,9,13$ & \\
\hline 97. & & Hypomecis punctinalis (Scopoli, 1763) & $1,3,9,14$ & \\
\hline 98. & & Hypomecis roboraria (Denis \& Schiffermüller, 1775) & $3,9,13,14$ & \\
\hline 99. & & Idaea aversata (Linnaeus, 1758) & $3,9,13,14,15,16$ & \\
\hline 100. & & Idaea biselata (Hufnagel, 1767) & 15,16 & - \\
\hline 101. & & Idaea degeneraria (Hübner, 1799) & 2,15 & \\
\hline 102. & & Idaea dimidiata (Hufnagel, 1767) & 9,13 & \\
\hline 103. & & Idaea moniliata (Denis \& Schiffermüller, 1775) & 15 & \\
\hline 104. & & Idaea muricata (Hufnagel, 1767) & 14,15 & \\
\hline 105. & & Idaea nitidata (Herrich-Schäffer, 1861) & 15 & \\
\hline 106. & & Idaea straminata (Borkhausen, 1794) & 15 & \\
\hline 107. & & Idaea trigeminata (Haworth, 1809) & 9,13 & \\
\hline 108. & & Lampropteryx suffumata (Denis \& Schiffermüller, 1775) & 14 & \\
\hline 109. & & Ligdia adustata (Denis \& Schiffermüller, 1775) & $\begin{array}{c}1,2,3,9,10,13,14 \\
15,16\end{array}$ & \\
\hline 110. & & Lobophora halterata (Hufnagel, 1767) & 2,14 & \\
\hline 111. & & Lomaspilis marginata (Linnaeus, 1758) & $\begin{array}{c}3,8,9,13,14,15 \\
16\end{array}$ & - \\
\hline 112. & & Lomographa bimaculata (Fabricius, 1775) & 14 & \\
\hline 113. & & Lomographa temerata (Denis \& Schiffermüller, 1775) & $9,14,15,16$ & \\
\hline 114. & & Macaria alternata (Denis \& Schiffermüller, 1775) & $3,9,13,14,15,16$ & \\
\hline 115. & & Macaria liturata $($ Clerck, 1759$)$ & 9,14 & \\
\hline 116. & & Melanthia procellata (Denis \& Schiffermüller, 1775) & $1,13,15$ & - \\
\hline 117. & & Mesoleuca albicillata (Linnaeus, 1758) & $2,8,9,13,16$ & $\bullet$ \\
\hline 118. & & Mesotype parallelolineata (Retzius, 1783) & 14 & \\
\hline 119. & & Minoa murinata (Scopoli, 1763) & 15 & \\
\hline 120. & & Opisthograptis luteolata (Linnaeus, 1758) & 2,14 & \\
\hline 121. & & Ourapteryx sambucaria (Linnaeus, 1758) & $13,14,15$ & \\
\hline 122. & & Paradarisa consonaria (Hübner, 1799) & 2 & \\
\hline 123. & & Parectropis similaria (Hufnagel, 1767) & 13,14 & \\
\hline 124. & & Pasiphila debiliata (Hübner, 1817) & 13 & \\
\hline 125. & & Peribatodes rhomboidaria (Denis \& Schiffermüller, 1775) & $\begin{array}{c}1,2,3,5,6,8,9,11 \\
12,13,14,15,16\end{array}$ & \\
\hline 126. & & Perizoma affinitata (Stephens, 1831) & & $\bullet$ \\
\hline 127. & & Perizoma alchemillata (Linnaeus, 1758) & $9,15,16$ & $\bullet$ \\
\hline 128. & & Perizoma flavofasciata (Thunberg, 1792) & 13,16 & \\
\hline 129. & & Perizoma lugdunaria (Herrich-Schäffer, 1855) & 9 & \\
\hline 130. & & Phileremevetulata (Denis \& Schiffermüller, 1775) & 14 & \\
\hline 131. & & Plagodis dolabraria (Linnaeus, 1767) & $9,14,15$ & \\
\hline 132. & & Plagodis pulveraria (Linnaeus, 1758) & 14 & $\bullet$ \\
\hline 133. & & Pseudopanthera macularia (Linnaeus, 1758) & 14 & \\
\hline 134. & & Pseudoterpna pruinata (Hufnagel, 1767) & $8,14,15$ & \\
\hline 135. & & Pungeleria capreolaria (Denis \& Schiffermüller, 1775) & $9,11,13,14$ & \\
\hline 136. & & Rhodostrophia vibicaria (Clerck, 1759) & 13,14 & \\
\hline 137. & & Scopula immorata (Linnaeus, 1758) & 15 & - \\
\hline 138. & & Scopula incanata (Linnaeus, 1758) & 15 & \\
\hline 139. & & Scopula nigropunctata (Hufnagel, 1767) & $5,9,15$ & \\
\hline
\end{tabular}




\begin{tabular}{|c|c|c|c|c|}
\hline & Family & Species & Locality & $\begin{array}{l}\text { Literature } \\
\text { records }\end{array}$ \\
\hline 140. & & Scopula ornata (Scopoli, 1763) & $2,14,15$ & \\
\hline 141. & & Scopula rubiginata (Hufnagel, 1767) & 15,16 & \\
\hline 142. & & Scopula virgulata (Denis \& Schiffermüller, 1775) & 15 & \\
\hline 143. & & Scotopteryx luridata (Hufnagel, 1767) & $2,14,15$ & \\
\hline 144. & & Selenia dentaria (Fabricius, 1775) & $10,14,16$ & \\
\hline 145. & & Selenia lunularia (Hübner, 1788) & 14 & $\bullet$ \\
\hline 146. & & Selenia tetralunaria (Hufnagel, 1767) & $9,10,14,15$ & \\
\hline 147. & & Spargania luctuata (Denis \& Schiffermüller, 1775) & & $\bullet$ \\
\hline 148. & & Stegania cararia (Hübner, 1790) & 2,15 & \\
\hline 149. & & Stegania dilectaria (Hübner, 1790) & 2 & \\
\hline 150. & & Thera variata (Denis \& Schiffermüller, 1775) & $9,14,15$ & $\bullet$ \\
\hline 151. & & Thera vetustata (Denis \& Schiffermüller, 1775) & 9 & \\
\hline 152. & & Timandra comae Schmidt, 1931 & 6,15 & \\
\hline 153. & & Trichopteryx carpinata (Borkhausen, 1794) & 2,1 & \\
\hline 154. & & Triphosa dubitata (Linnaeus, 1758) & 10,14 & \\
\hline 155. & & Xanthorhoe biriviata (Borkhausen, 1794) & 16 & $\bullet$ \\
\hline 156. & & Xanthorhoe ferrugata (Clerck, 1759) & $3,14,16$ & \\
\hline 157. & & Xanthorhoe montanata (Denis \& Schiffermüller, 1775) & & $\bullet$ \\
\hline 158. & & Xanthorhoe quadrifasiata (Clerck, 1759) & 16 & \\
\hline 159. & Oecophoridae & Alabonia staintoniella (Zeller, 1850) & 13 & \\
\hline 160. & Chiambachidae & Diurnea fagella (Denis \& Schiffermüller, 1775) & 2,1 & \\
\hline 161. & Elachistidae & Agonopterix arenella (Denis \& Schiffermüller, 1775) & 14 & \\
\hline 162. & & Agonopterix ciliella (Stainton, 1849 ) & 14 & \\
\hline 163. & & Agonopterix nervosa (Haworth, 1811) & 14 & \\
\hline 164. & & Agonopterix propinquella (Treitschke, 1835) & 8 & \\
\hline 165. & & Hypercallia citrinalis (Scopoli, 1763) & 14 & \\
\hline 166. & & $\begin{array}{l}\text { Semioscopis steinkellneriana (Denis \& Schiffermüller, } \\
\text { 1775) }\end{array}$ & 14 & \\
\hline 167. & Hepialidae & Triodia sylvina (Linnaeus, 1761) & $2,6,8,15$ & \\
\hline 168. & Lasiocampidae & Dendrolimus pini (Linnaeus, 1758) & 9 & \\
\hline 169. & & Gastropacha quercifolia (Linnaeus, 1758) & 14 & \\
\hline 170. & & Lasiocampa quercus (Linnaeus, 1758) & 9 & $\bullet$ \\
\hline 171. & & Macrothylacia rubi (Linnaeus, 1758) & 16 & \\
\hline 172. & & Malacosoma castrensis (Linnaeus, 1758) & 14 & \\
\hline 173. & & Odonestis pruni (Linnaeus, 1758) & 14 & \\
\hline 174. & & Phyllodesma tremulifolia (Hübner, 1810) & 16 & \\
\hline 175. & & Poecilocampa populi (Linnaeus, 1758) & 14 & \\
\hline 176. & Erebidae & Amata phegea (Linnaeus, 1758) & 16 & $\bullet$ \\
\hline 177. & & Arctia caja (Linnaeus, 1758) & & $\bullet$ \\
\hline 178. & & Arctia villica (Linnaeus, 1758) & $9,13,14$ & $\bullet$ \\
\hline 179. & & Arctornis l-nigrum (Muller, 1764) & 15 & $\bullet$ \\
\hline 180. & & Atolmis rubricollis (Linnaeus, 1758) & 9 & \\
\hline 181. & & Calliteara pudibunda (Linnaeus, 1758) & 3,8 & $\bullet$ \\
\hline 182. & & Callimorpha dominula (Linnaeus, 1758) & 14 & $\bullet$ \\
\hline 183. & & Calyptra thalictri (Borkhausen, 1790) & 9 & \\
\hline 184. & & Catocala elocata (Esper, 1787) & & $\bullet$ \\
\hline 185. & & Catocala fulminea (Scopoli, 1763) & 15 & \\
\hline 186. & & Catocala nupta (Linnaeus, 1767) & 15 & $\bullet$ \\
\hline 187. & & Catocala promissa (Denis \& Schiffermüller, 1775) & 15 & \\
\hline
\end{tabular}




\begin{tabular}{|c|c|c|c|c|}
\hline & Family & Species & Locality & $\begin{array}{l}\text { Literature } \\
\text { records }\end{array}$ \\
\hline 188. & & Catocala sponsa (Linnaeus, 1767) & 15 & \\
\hline 189. & & Cybosia mesomella (Linnaeus, 1758) & 9,13 & $\bullet$ \\
\hline 190. & & Dysauxes ancilla (Linnaeus, 1767) & 15,16 & - \\
\hline 191. & & Dysgonia algira (Linnaeus, 1767) & $2,5,15$ & \\
\hline 192. & & Eilema caniola (Hübner, 1808) & 9,15 & \\
\hline 193. & & Eilema complana (Linnaeus, 1758) & 13,14 & \\
\hline 194. & & Eilema depressa (Esper, 1787) & $8,9,13,15$ & \\
\hline 195. & & Eilema lurideola (Zincken, 1817) & 14 & \\
\hline 196. & & Eilema sororcula (Hufnagel, 1766) & $2,9,10,13,14,16$ & \\
\hline 197. & & Euclidia glyphica (Linnaeus, 1758) & 4,15 & \\
\hline 198. & & Euclidia mi (Clerck, 1759) & 15 & \\
\hline 199. & & Euplagia quadripunctaria (Poda, 1761) & $4,7,8,9,15,16$ & $\bullet$ \\
\hline 200. & & Euproctis similis (Fuessly, 1775) & 9 & \\
\hline 201. & & Herminia grisealis (Denis \& Schiffermüller, 1775) & $2,8,9,13,15,16$ & \\
\hline 202. & & Herminia tarsicrinalis (Knoch, 1782) & $\begin{array}{c}1,3,8,9,13,14,15 \\
16\end{array}$ & \\
\hline 203. & & Herminia tarsipennalis (Treitschke, 1835) & 8,15 & \\
\hline 204. & & Herminia tenuialis (Rebel, 1899) & 16 & \\
\hline 205. & & Hypena proboscidalis (Linnaeus, 1758) & $2,3,7,8,12,13$ & $\bullet$ \\
\hline 206. & & Hypena rostralis (Linnaeus, 1758) & 2 & \\
\hline 207. & & Idia calvaria (Denis \& Schiffermüller, 1775) & 3 & \\
\hline 208. & & Laspeyria flexula (Denis \& Schiffermüller, 1775) & $9,13,14,15$ & \\
\hline 209. & & Lithosia quadra (Linnaeus, 1758) & $2,8,9,13,14,15$ & \\
\hline 210. & & Lygephila craccae (Denis \& Schiffermüller, 1775) & 14,15 & \\
\hline 211. & & Lygephila pastinum (Treitschke, 1826) & 16 & \\
\hline 212. & & Lymantria dispar (Linnaeus, 1758) & $9,15,16$ & \\
\hline 213. & & Lymantria monacha (Linnaeus, 1758) & 15,16 & $\bullet$ \\
\hline 214. & & Metachrostis velox (Hübner, 1813) & 15 & \\
\hline 215. & & Miltochrista miniata (Forster, 1771) & $8,9,13$ & - \\
\hline 216. & & Paracolax tristalis (Fabricius, 1794) & $9,14,15$ & \\
\hline 217. & & Pechipogo strigilata (Linnaeus, 1758) & $9,13,16$ & \\
\hline 218. & & Phragmatobia fuliginosa (Linnaeus, 1758) & $8,14,15,16$ & \\
\hline 219. & & Phytometra viridaria (Clerck, 1759) & 14,15 & \\
\hline 220. & & Polypogon gryphalis (Herrich-Schäffer, 1851) & 15 & \\
\hline 221. & & Polypogon tentacularia (Linnaeus, 1758) & 13 & \\
\hline 222. & & Rivula sericealis (Scopoli, 1763) & $2,3,4,8,11,15,16$ & \\
\hline 223. & & Scoliopteryx libatrix (Linnaeus, 1758) & & $\bullet$ \\
\hline 224. & & Schrankia costaestrigalis (Stephens, 1834) & 5,8 & \\
\hline 225. & & Spilosoma lubricipeda (Linnaeus, 1758) & 3,9 & \\
\hline 226. & & Spilosoma lutea (Hufnagel, 1766) & 1 & \\
\hline 227. & & Trisateles emortualis (Denis \& Schiffermüller, 1775) & $9,13,15$ & - \\
\hline 228. & & Zanclognatha lunalis (Scopoli, 1763) & & $\bullet$ \\
\hline 229. & Noctuidae & Abrostola triplasia (Linnaeus, 1758) & 2 & \\
\hline 230. & & Acontia candefacta (Hubner, 1831) & 15 & \\
\hline 231. & & Acontia trabealis (Scopoli, 1763) & $3,14,15$ & \\
\hline 232. & & Acronicta aceris (Linnaeus, 1758) & 14,15 & \\
\hline 233. & & Acronicta alni (Linnaeus, 1767) & 14 & \\
\hline 234. & & Acronicta cuspis (Hübner, 1813)* & 15 & \\
\hline 235. & & Acronicta euphorbiae (Denis \& Schiffermüller, 1775) & 14 & \\
\hline
\end{tabular}




\begin{tabular}{|c|c|c|c|c|}
\hline & Family & Species & Locality & $\begin{array}{l}\text { Literature } \\
\text { records }\end{array}$ \\
\hline 236. & & Acronicta psi (Linnaeus, 1758$)^{*}$ & 15 & \\
\hline 237. & & Acronicta rumicis (Linnaeus, 1758) & $2,8,14,15$ & \\
\hline 238. & & Aedia leucomelas (Linnaeus, 1758) & $3,8,15,16$ & \\
\hline 239. & & Agrochola helvola (Linnaeus, 1758) & 15 & \\
\hline 240. & & Agrochola litura (Linnaeus, 1758) & 14 & \\
\hline 241. & & Agrochola macilenta (Hübner, 1809) & 15 & \\
\hline 242. & & Agrotis exclamationis (Linnaeus, 1758) & $3,13,14$ & \\
\hline 243. & & Agrotis ipsilon (Hufnagel, 1766) & 13,14 & \\
\hline 244. & & Agrotis segetum (Denis \& Schiffermüller, 1775) & $12,13,16$ & \\
\hline 245. & & Allophyes oxyacanthae (Linnaeus, 1758) & $9,14,15$ & \\
\hline 246. & & Ammосопіа саесітасиla (Denis \& Schiffermüller, 1775) & 15 & \\
\hline 247. & & Amphipyra livida (Denis \& Schiffermüller, 1775) & 15 & \\
\hline 248. & & Amphipyra pyramidea (Linnaeus, 1758$)^{*}$ & $2,14,15$ & \\
\hline 249. & & Amphipyra tragopoginis (Clerck, 1759) & & - \\
\hline 250. & & Anorthoa munda (Denis \& Schiffermüller, 1775) & 2 & \\
\hline 251. & & Apamea monoglypha (Hufnagel, 1766) & 3 & $\bullet$ \\
\hline 252. & & Apamea scolopacina (Esper, 1788) & 15 & \\
\hline 253. & & Athetis lepigone (Möschler, 1860, 1860) & $5,8,9,15$ & \\
\hline 254. & & Atypha pulmonaris (Esper, 1790) & $9,13,15$ & \\
\hline 255. & & Autographa gamma (Linnaeus, 1758) & $2,4,8,14,15,16$ & \\
\hline 256. & & Autographa jota (Linnaeus, 1758) & & $\bullet$ \\
\hline 257. & & Autographa pulchrina (Haworth, 1809) & & $\bullet$ \\
\hline 258. & & Axylia putris (Linnaeus, 1761) & 14 & \\
\hline 259. & & Callopistria juventina (Stoll, 1782) & 9 & \\
\hline 260. & & Cerastis rubricosa (Denis \& Schiffermüller, 1775) & 2,14 & \\
\hline 261. & & Colocasia coryli (Linnaeus, 1758) & $1,2,3,9,10,14,16$ & \\
\hline 262. & & Conistra erythrocephala (Denis \& Schiffermüller, 1775) & 14 & \\
\hline 263. & & Conistra rubiginea (Denis \& Schiffermüller, 1775) & $2,10,14$ & \\
\hline 264. & & Conistra rubiginosa (Scopoli, 1763) & 14 & \\
\hline 265. & & Conistra vaccinii (Linnaeus, 1761) & $2,9,14$ & \\
\hline 266. & & Cosmia trapezina (Linnaeus, 1758$)$ & $8,15,16$ & \\
\hline 267. & & Craniophora ligustri (Denis \& Schiffermüller, 1775) & $2,8,9,14,15$ & \\
\hline 268. & & Cryphia algae (Fabricius, 1775$)^{*}$ & $14,15,16$ & \\
\hline 269. & & Cryphia receptricula (Hübner, 1803)* & 14 & \\
\hline 270. & & Deltote bankiana (Fabricius, 1775 ) & 13 & \\
\hline 271. & & Deltote pygarga (Hufnagel, 1766) & $13,14,15$ & \\
\hline 272. & & Diachrysia chrysitis (Linnaeus, 1758) & 2 & \\
\hline 273. & & Diloba caeruleocephala (Linnaeus, 1758) & 14,15 & \\
\hline 274. & & Dypterygia scabriuscula (Linnaeus, 1758) & 13 & \\
\hline 275. & & Egira conspicillaris (Linnaeus, 1758) & 14 & \\
\hline 276. & & Elaphria venustula (Hübner, 1790) & 16 & \\
\hline 277. & & Epilecta linogrisea (Denis \& Schiffermüller, 1775) & 8,15 & \\
\hline 278. & & Eugnorisma depuncta (Linnaeus, 1761) & $5,6,8,9,12,15$ & \\
\hline 279. & & Euplexia lucipara (Linnaeus, 1758) & 9,16 & \\
\hline 280. & & Gortyna flavago (Denis \& Schiffermüller, 1775) & 14 & \\
\hline 281. & & Griposia aprilina (Linnaeus, 1758) & 15 & \\
\hline 282. & & Helicoverpa armigera (Hübner, 1808) & $5,8,14,15$ & \\
\hline 283. & & Hoplodrina ambigua (Denis \& Schiffermüller, 1775) & 15 & \\
\hline 284. & & Lacanobia oleracea (Linnaeus, 1758) & 8,13 & \\
\hline
\end{tabular}




\begin{tabular}{|c|c|c|c|c|}
\hline & Family & Species & Locality & $\begin{array}{l}\text { Literature } \\
\text { records }\end{array}$ \\
\hline 285. & & Lamprosticta culta (Denis \& Schiffermüller, 1775) & 15 & \\
\hline 286. & & Leucania comma (Linnaeus, 1761) & & - \\
\hline 287. & & Lithophane ornitopus (Hufnagel, 1766) & 2 & \\
\hline 288. & & Lithophane socia (Hufnagel, 1766) & 2 & \\
\hline 289. & & Luperina dumerilii (Duponchel, 1826) & 2 & \\
\hline 290. & & Mesoligia furuncula (Denis \& Schiffermüller, 1775) & 15 & \\
\hline 291. & & Mniotype satura (Denis \& Schiffermüller, 1775) & 14 & \\
\hline 292. & & Moma alpium (Osbeck, 1778) & 15,16 & \\
\hline 293. & & Mormo maura (Linnaeus, 1758) & 8 & $\bullet$ \\
\hline 294. & & Mythimna albipuncta (Denis \& Schiffermüller, 1775) & $8,14,15$ & \\
\hline 295. & & Mythimna congrua (Hübner, 1817) & 8 & \\
\hline 296. & & Mythimna ferrago (Fabricius, 1787) & $5,14,15,16$ & \\
\hline 297. & & Mythimna l-album (Linnaeus, 1767) & 9,15 & \\
\hline 298. & & Mythimna turca (Linnaeus, 1761) & 3 & \\
\hline 299. & & Noctua comes Hübner, 1813 & $\begin{array}{c}2,5,8,11,12,14 \\
15\end{array}$ & \\
\hline 300. & & Noctua fimbriata (Schreber, 1759$)^{*}$ & $2,8,13,15$ & \\
\hline 301. & & Noctua interposita (Hübner, 1790) & 15 & \\
\hline 302. & & Noctua janthina Denis \& Schiffermüller, 1775 & $2,8,15$ & \\
\hline 303. & & Noctua pronuba (Linnaeus, 1758) & $\begin{array}{c}2,3,5,8,9,12,13 \\
14,15\end{array}$ & $\bullet$ \\
\hline 304. & & Ochropleura plecta (Linnaeus, 1761) & 3,14 & \\
\hline 305. & & Oligia latruncula (Denis \& Schiffermüller, 1775)* & 8,13 & \\
\hline 306. & & Oligia strigilis (Linnaeus, 1758)* & 13,14 & \\
\hline 307. & & Orthosia cerasi (Fabricius, 1775) & 2,14 & \\
\hline 308. & & Orthosia cruda (Denis \& Schiffermüller, 1775) & 2 & \\
\hline 309. & & Orthosia gothica (Linnaeus, 1758) & 2,14 & \\
\hline 310. & & Orthosia gracilis (Denis \& Schiffermüller, 1775) & 14 & \\
\hline 311. & & Orthosia incerta (Hufnagel, 1766) & 2,14 & \\
\hline 312. & & Orthosia populeti (Fabricius, 1775) & 2 & \\
\hline 313. & & Phlogophora meticulosa (Linnaeus, 1758) & 15 & \\
\hline 314. & & Polia bombycina (Hufnagel, 1766) & 13 & \\
\hline 315. & & Polyphaenis sericata (Esper, 1787) & 15 & \\
\hline 316. & & Pyrrhia umbra (Hufnagel, 1766) & 9,14 & \\
\hline 317. & & Sideridis rivularis (Fabricius, 1775) & 15 & \\
\hline 318. & & Thalpophila matura (Hufnagel, 1766) & 14 & \\
\hline 319. & & Tholera cespitis (Denis \& Schiffermüller, 1775) & 15 & \\
\hline 320. & & Tiliacea aurago (Denis \& Schiffermüller, 1775) & 14,15 & \\
\hline 321. & & Tiliacea citrago (Linnaeus, 1758) & 14 & \\
\hline 322. & & Tiliacea sulphurago (Denis \& Schiffermüller, 1775) & 14 & \\
\hline 323. & & Trachea atriplicis (Linnaeus, 1758) & 9 & \\
\hline 324. & & Valeria oleagina (Denis \& Schiffermüller, 1775) & 2 & \\
\hline 325. & & Xanthia icteritia (Hufnagel, 1766) & 15 & \\
\hline 326. & & Xestia castanea (Esper, 1798) & 14 & \\
\hline 327. & & Xestia c-nigrum (Linnaeus, 1758) & $2,3,14,15$ & \\
\hline 328. & & Xestia ditrapezium (Denis \& Schiffermüller, 1775) & 13 & \\
\hline 329. & & Xestia stigmatica (Hübner, 1813) & 8,15 & \\
\hline 330. & & Xestia triangulum (Hufnagel, 1766) & 15 & \\
\hline 331. & & Xestia xanthographa (Denis \& Schiffermüller, 1775) & $2,6,8,14,15$ & \\
\hline
\end{tabular}




\begin{tabular}{|c|c|c|c|c|}
\hline & Family & Species & Locality & $\begin{array}{l}\text { Literature } \\
\text { records }\end{array}$ \\
\hline 332. & Nolidae & Bena bicolorana (Fuessly, 1775) & 15 & \\
\hline 333. & & Earias vernana (Fabricius, 1787) & 15 & \\
\hline 334. & & Meganola albula (Denis \& Schiffermüller, 1775) & $8,9,15$ & \\
\hline 335. & & Meganola strigula (Denis \& Schiffermüller, 1775) & 14,15 & \\
\hline 336. & & Meganola togatulalis (Hübner, 1796) & 14 & \\
\hline 337. & & Nola cicatricalis (Treitschke, 1835) & 14 & \\
\hline 338. & & Nola cristatula (Hübner, 1793) & 10 & \\
\hline 339. & & Pseudoips prasinana (Linnaeus, 1758 ) & $8,9,10,14,15,16$ & \\
\hline 340. & Notodontidae & Clostera anachoreta (Denis \& Schiffermüller, 1775) & 14 & \\
\hline 341. & & Clostera curtula (Linnaeus, 1758) & 14,16 & $\bullet$ \\
\hline 342. & & Clostera pigra (Hufnagel, 1766) & 14 & \\
\hline 343. & & Drymonia melagona (Borkhausen, 1790) & $9,13,15,16$ & $\bullet$ \\
\hline 344. & & Drymonia ruficornis (Hufnagel, 1766) & 14 & \\
\hline 345. & & Drymonia velitaris (Hufnagel, 1766) & 14 & \\
\hline 346. & & Furcula furcula (Clerck, 1759) & 14 & \\
\hline 347. & & Gluphisia crenata (Esper, 1785) & 14,16 & \\
\hline 348. & & Harpyia milhauseri (Fabricius, 1775) & 14 & \\
\hline 349. & & Notodonta dromedarius (Linnaeus, 1767) & 8,15 & \\
\hline 350. & & Notodonta tritophus (Denis \& Schiffermüller, 1775) & 13 & $\bullet$ \\
\hline 351. & & Notodonta ziczac (Linnaeus, 1758) & 3 & \\
\hline 352. & & Peridea anceps (Goeze, 1781) & 14 & \\
\hline 353. & & Phalera bucephaloides (Ochsenheimer, 1810) & 15 & \\
\hline 354. & & Pheosia tremula (Clerck, 1759) & 2 & \\
\hline 355. & & Ptilodon capucina (Linnaeus, 1758) & $3,9,13$ & \\
\hline 356. & & Ptilodon cucullina (Denis \& Schiffermüller, 1775) & $8,9,13,14,15,16$ & - \\
\hline 357. & & Ptilophora plumigera (Denis \& Schiffermüller, 1775) & 14 & \\
\hline 358. & & Spatalia argentina (Denis \& Schiffermüller, 1775) & $3,14,15,16$ & \\
\hline 359. & & Stauropus fagi (Linnaeus, 1758) & $9,13,14,15,16$ & \\
\hline 360. & Crambidae & Agrotera nemoralis (Scopoli, 1763) & $3,9,13,14$ & \\
\hline 361. & & Anania coronata (Hufnagel, 1767) & 13 & \\
\hline 362. & & Anania crocealis (Hübner, 1796) & 14,15 & \\
\hline 363. & & Anania fuscalis (Denis \& Schiffermüller, 1775) & 15 & \\
\hline 364. & & Anania hortulata (Linnaeus, 1758) & 13,16 & \\
\hline 365. & & Anania lancealis (Denis \& Schiffermüller, 1775) & 13 & \\
\hline 366. & & Anania stachydalis (Germar, 1821) & $8,9,13,14,15$ & \\
\hline 367. & & Anania terrealis (Treitschke, 1829) & $5,14,15$ & \\
\hline 368. & & Anania verbascalis (Denis \& Schiffermüller, 1775) & $8,9,14,15$ & \\
\hline 369. & & Catoptria falsella (Denis \& Schiffermüller, 1775) & 15 & \\
\hline 370. & & Catoptria osthelderi (Lattin, 1950)* & 15 & \\
\hline 371. & & Catoptria pinella (Linnaeus, 1758$)^{*}$ & 15 & \\
\hline 372. & & Chrysocrambus linetella (Fabricius, 1781) & 16 & \\
\hline 373. & & Chrysoteuchia culmella (Linnaeus, 1758) & 15 & \\
\hline 374. & & Crambus lathoniellus (Zincken, 1817) & 16 & \\
\hline 375. & & Crambus pascuella (Linnaeus, 1758) & 13 & \\
\hline 376. & & Crambus perlella (Scopoli, 1763) & 15 & \\
\hline 377. & & Cydalima perspectalis (Walker, 1859) & $8,14,15$ & \\
\hline 378. & & Cynaeda dentalis (Denis \& Schiffermüller, 1775) & 2,15 & \\
\hline 379. & & Diasemiopsis ramburialis (Duponchel, 1834) & 8 & \\
\hline 380. & & Dolicharthria punctalis (Denis \& Schiffermüller, 1775) & 2,15 & \\
\hline
\end{tabular}




\begin{tabular}{|c|c|c|c|c|}
\hline & Family & Species & Locality & $\begin{array}{l}\text { Literature } \\
\text { records }\end{array}$ \\
\hline 381. & & Ecpyrrhorrhoe rubiginalis (Hübner, 1796) & 8 & \\
\hline 382. & & Evergestis forficalis (Linnaeus, 1758) & 13 & \\
\hline 383. & & Evergestis limbata (Linnaeus, 1767) & 13 & \\
\hline 384. & & Evergestis pallidata (Hufnagel, 1767) & $1,8,9,13$ & \\
\hline 385. & & Metasia ophialis (Treitschke, 1829) & $2,15,16$ & \\
\hline 386. & & Nascia cilialis (Hübner, 1796) & 16 & \\
\hline 387. & & Nomophila noctuella (Denis \& Schiffermüller, 1775) & 2,8 & \\
\hline 388. & & Ostrinia nubilalis (Hübner, 1796) & $9,13,16$ & \\
\hline 389. & & Palpita vitrealis (Rossi, 1794) & 14 & \\
\hline 390. & & Pediasia contaminella (Hübner, 1796) & $2,8,15$ & \\
\hline 391. & & Pleuroptya ruralis (Scopoli, 1763) & $2,5,6,8,9,14$ & \\
\hline 392. & & Pyrausta aurata (Scopoli, 1763) & 8,15 & \\
\hline 393. & & Pyrausta cingulata (Linnaeus, 1758) & 15 & \\
\hline 394. & & Pyrausta purpuralis (Linnaeus, 1758) & 14,15 & \\
\hline 395. & & Sitochroa palealis (Denis \& Schiffermüller, 1775) & 9 & \\
\hline 396. & & Sitochroa verticalis (Linnaeus, 1758) & 14 & \\
\hline 397. & & Udea ferrugalis (Hübner, 1796) & $14,15,16$ & \\
\hline 398. & & Udea olivalis (Denis \& Schiffermüller, 1775) & 15 & \\
\hline 399. & & Xanthocrambus lucellus (Herrich-Schäffer, 1848) & 15 & \\
\hline 400. & Pyralidae & Aphomia sociella (Linnaeus, 1758) & 14 & \\
\hline 401. & & Dioryctria abietella (Denis \& Schiffermüller, 1775) & 14 & \\
\hline 402. & & Endotricha flammealis (Denis \& Schiffermüller, 1775) & $8,15,16$ & \\
\hline 403. & & Homoeosoma sinuella (Fabricius, 1794) & 14 & \\
\hline 404. & & Hypochalcia propinquella (Guenée, 1845) & 14 & \\
\hline 405. & & Hypsopygia costalis (Fabricius, 1775) & $8,9,14,15$ & \\
\hline 406. & & Hypsopygia glaucinalis (Linnaeus, 1758) & 6,8 & \\
\hline 407. & & Hypsopygia rubidalis (Denis \& Schiffermüller, 1775) & 16 & \\
\hline 408. & & Myelois circumvoluta (Fourcroy, 1785) & 16 & \\
\hline 409. & & Oncocera semirubella (Scopoli, 1763) & $8,14,15$ & \\
\hline 410. & & Pyralis farinalis (Linnaeus, 1758) & 14,15 & \\
\hline 411. & & Pyralis regalis Denis \& Schiffermüller, 1775 & 15 & \\
\hline 412. & Pterophoridae & $\begin{array}{l}\text { Cnaemidophorus rhododactyla (Denis \& Schiffermüller, } \\
\text { 1775) }\end{array}$ & $14,15,16$ & \\
\hline 413. & & Emmelina monodactyla (Linnaeus, 1758) & 14 & \\
\hline 414. & & Platyptilia farfarellus Zeller, $1867^{*}$ & 16 & \\
\hline 415. & Psychidae & Bijugis bombycella (Denis \& Schiffermüller, 1775) & 14,16 & \\
\hline 416. & Tortricidae & Acleris cristana (Denis \& Schiffermüller, 1775) & 8 & \\
\hline 417. & & Acleris laterana (Fabricius, 1794) & 12 & \\
\hline 418. & & Acleris variegana (Denis \& Schiffermüller, 1775) & 14,15 & \\
\hline 419. & & Agapeta zoegana (Linnaeus, 1767) & 15 & \\
\hline 420. & & Aleimma loeflingiana (Linnaeus, 1758) & 2,14 & \\
\hline 421. & & Ancylis badiana (Denis \& Schiffermüller, 1775) & 8 & \\
\hline 422. & & Ancylis mitterbacheriana (Denis \& Schiffermüller, 1775) & 16 & \\
\hline 423. & & Archips podana (Scopoli, 1763) & $8,13,14,15$ & \\
\hline 424. & & Archips xylosteana (Linnaeus, 1758) & 14 & \\
\hline 425. & & Clepsis rurinana (Linnaeus, 1758) & 16 & \\
\hline 426. & & Cochylis hybridella (Hübner, 1813) & 14 & \\
\hline 427. & & Cydia pomonella (Linnaeus, 1758) & $8,14,15$ & \\
\hline 428. & & Endothenia marginana (Haworth, 1811) & 14 & \\
\hline
\end{tabular}




\begin{tabular}{|c|c|c|c|c|}
\hline & Family & Species & Locality & $\begin{array}{l}\text { Literature } \\
\text { records }\end{array}$ \\
\hline 429. & & Endothenia quadrimaculana (Haworth, 1811) & $3,8,14$ & \\
\hline 430. & & Epinotia festivana (Hübner, 1799) & 14 & \\
\hline 431. & & Eucosma cana (Haworth, 1811) & 15 & \\
\hline 432. & & Grapholita fissana (Frölich, 1828) & 14 & \\
\hline 433. & & Gravitarmata margarotana (Heinemann, 1863) & 14 & \\
\hline 434. & & Gypsonoma sociana (Haworth, 1811) & 14,15 & \\
\hline 435. & & Hedya nubiferana (Haworth, 1811) & 14 & \\
\hline 436. & & Hedya salicella (Linnaeus, 1758) & 16 & \\
\hline 437. & & Metendothenia atropunctana (Zetterstedt, 1839) & 14 & \\
\hline 438. & & Notocelia incarnatana (Hübner, 1800) & 15 & \\
\hline 439. & & Notocelia uddmanniana (Linnaeus, 1758) & 8 & \\
\hline 440. & & Pammene trauniana (Denis \& Schiffermüller, 1775) & 1 & \\
\hline 441. & & Pandemis corylana (Fabricius, 1794) & 1 & \\
\hline 442. & & Paramesia gnomana (Clerck, 1759) & 14 & \\
\hline 443. & & Philedone gerningana (Denis \& Schiffermüller, 1775) & 15 & \\
\hline 444. & & Phtheochroa annae Huemer, 1990 & 14 & \\
\hline 445. & & Pseudeulia asinana (Hübner, 1799) & 14 & \\
\hline 446. & & Rhyacionia buoliana (Denis \& Schiffermüller, 1775) & 15 & \\
\hline 447. & & Spilonota ocellana (Denis \& Schiffermüller, 1775) & 14 & \\
\hline 448. & & Tortrix viridana Linnaeus, 1758 & 14 & \\
\hline 449. & Limacodidae & Apoda limacodes (Hufnagel, 1766) & $9,15,16$ & $\bullet$ \\
\hline 450. & & Heterogenea asella (Denis \& Schiffermüller, 1775) & 9,16 & \\
\hline 451. & \begin{tabular}{|l|l} 
Plutellidae \\
\end{tabular} & Plutella xylostella (Linnaeus, 1758) & 14 & \\
\hline 452. & Yponomeutidae & Yponomeuta padella (Linnaeus, 1758) & 15 & \\
\hline 453. & & Yponomeuta plumbella (Denis \& Schiffermüller, 1775) & 15 & \\
\hline 454. & Zygaenidae & Zygaena carniolica (Scopoli, 1763) & 14 & $\bullet$ \\
\hline 455. & & Zygaena transalpina (Esper, 1780) & & $\bullet$ \\
\hline
\end{tabular}

*Species for which the genitals were checked to confirm the correct identification.

\section{DISCUSSION}

With 437 species recorded during this survey, the area of Medvednica Natur Park can now be regarded as one of the better surveyed areas of Croatia with respect to the moth fauna (e.g. Kranjčev, 1985; Habeler, 2008; Vignjević et al., 2010; Koren \& LadAvAC, 2013). Also, it is the only nature park in Croatia of the 11 existing ones, aside from Kopački Rit, only a part of the moth fauna of which was studied (Vignjević et al., 2010), to have a checklist of moths. This is certainly not the final number of species, but probably only $40-50 \%$ of the estimated moth fauna species of Medvednica Nature Park. Future surveys, as well the study of Microlepidoptera families will certainly increase the confirmed number of species in the area. The data from this survey can be used for the better management of the habitats as well for promotion and awareness-raising of the local communities and visitors of the Nature Park. 
Checklists for most moth families in Croatia and Red Lists are still missing, therefore is difficult to put our results in a meaningful perspective regarding the species' rarity or threat status. Thus, the species' status can only be subjectively evaluated which may lead to the wrong conclusions. In today's drastic changes in land use, it is important to preserve habitat richness for moths in the Nature Park. Although the Nature Park is mostly forested, meadows and pastures as well as the plant-rich forest edge are very important for the long-term survival of moths. The remaining meadows are nowadays found mainly in the marginal parts of the Park (eg Vejalnica, Bizek, Golubovec ponds). It is at these sites that the butterfly fauna is very diverse and rich. However, it should be noted that abandonment of meadows has been observed, especially in the area of Vejalnica. Most meadows nowadays are no longer mowed or grazed, which results in the disappearance of many plant and animal species from the area. Some grassland species reported in the literature, like Z. transalpina, were not confirmed during our survey. As members of the family Zagaenidae are known to be sensitive to the changes in use of meadows, this may indicate a negative change in grassland diversity. However targeted surveys of day-flying Zygaenidae of the Nature park is needed in order to assess the status of this family in the area.

During this survey, habitat succession was observed in almost all grassland habitats. For this reason, habitat remediation actions are required, like removing shrubby vegetation from the meadows and starting regular mowing. The central, forested part of Medvednica belongs to less favourable habitats for moths, as there is mainly dense forest. The meadows that still exist in that part are intensely mowed, and as such not suitable for the survival of either caterpillar plants or adult moths. To preserve fauna diversity in the area, meadows should be recovered and extensively maintained, to preserve host plants for larvae and flowers as nectar source for the adults.

Also important is the connectivity of the fragmented grassland habitats across Medvednica NP. In a survey in the deciduous forests of eastern North America, increased forest fragmentation was found to change moth community composition as a result of both species impoverishment and replacement (SCHмidT \& Roland, 2009). In order to accomplish that, forest edges should be maintained open in order to provide corridors to vagrant moths and other animal species.

\section{ACKNOWLEDGMENTS}

I would like to thank the Public Institution Medvednica Nature Park for recognizing the value of this project and financially supporting it. I am grateful to Peter Hüemer for the confirmation of the correct identification of $P$. annae. My heartfelt thanks also go to my colleagues at the Hyla Association for their selfless assistance during field trips. I am also in debt to two anonymous reviewers for critical reviews and useful comments on the manuscript.

Received February 16, 2020

\section{REFERENCES}

de Jong, Y., Verbeek, M., Michelsen, V., Bjørn, P. de P., Los, W., Steeman, F., Bailly, N., Basire, C., Chylarecki, P., Stloukal, E., Hagedorn, G., Wetzel, F., Glöckler, F., Kroupa, A., Korb, G., Hoffmann, A., Häuser, C., Kohlbecker, A., Müller, A., Güntsch, A., Stoev, P. \& Penev, L., 2014: Fauna Europaea - all European animal species on the web. Biodiversity Data Journal 2, 1-35. 
Dobrović, I., Nikolić, T., Jelaska, S., Plazibat, M., Hršak, V. \& Šoštarić, R., 2006: An evaluation of floristic diversity in Medvednica Nature Park (northwestern Croatia). Plant Biosystems 140, 234-244.

Fibiger, M., 1997: Noctuidae Europaeae. Vol. 3 (Noctuinae III). Entomological Press, Sorø, 418 pp.

GRund, A., 1916: Beiträge zur kroatischen Lepidopteren-Fauna. Glasnik Hrvatskog prirodoslovnog društva 28, 95-105 \& 114-168.

Habeler, H., 2008[2003]: Die Schmetterlinge der Adria-Insel Krk - eine ökofaunistische Studie. Esperiana - Buchreihe zur Entomologie, 221 pp.

HAHN, M. \& BRÜHL, A. C., 2016: The secret pollinators: an overview of moth pollination with a focus on Europe and North America. Arthropod-Plant Interactions 10(1), 21-28.

Hausmann, A. \& ViIdalepp, J., 2012: Geometrid Moths of Europe, 3. Larentiinae 1. Apollo Books, Denmark, 743 pp.

HüEmer, P., 1990: Phtheochroa rugosana auct. - a species complex (Lepidoptera: Tortricidae). Nota Lepidopterologica 12(4), 269-289.

Koren, T. \& Ladavac, LJ., 2013: Diversity of Macroheterocera (except fam. Noctuidae [sensu novo] and fam. Geometridae) of central Istria, Croatia. Natura Croatica 22, 73-94.

Koren, T., Zadravec, M., ŠTiH, A., Burić, I., Lauš, B., 2018: The rediscovered Lepidoptera collection of Vanda Kochansky - Devidé. Acta entomologica slovenica 26, 63-74.

KRANJČEV, R., 1985: Odnos faune makrolepidoptera prema prirodnim i antropogenim staništima Podravine i podravskih pijesaka (I). Podravski zbornik 11, 200-226.

Kristensen, N., Scoble, M. \& Karsholt, O., 2006: Lepidoptera phylogeny and systematics: The state of inventorying moth and butterfly diversity. Zootaxa 1668, 699-747.

Kučinić, M., Hrašovec, B., 1999: Faunal and zoogeographical review of the Lepidoptera collection of the Faculty of Forestry, University of Zagreb, Part I: Noctuidae (Insecta: Lepidoptera). Natura Croatica 8(1): 27-47.

MaceK, J., Dvořák, J., Traxler, L. \& Červenka, V., 2008: Noční motýli II. Motýli a housenky střední Evropy - můrovití. Academia, Praha, 490 pp.

Macek, J., ProcházKa, J. \& Traxler, L., 2012: Noční motýli III. - píd’alkovití. Motýli a housenky střední Evropy. Academia, Praha, 424 pp.

Matošević, D., Pernek, M., Dubravac, T. \& Barić, B., 2009: Istraživanje faune lisnih minera drvenastog bilja u Hrvatskoj. Šumarski list 133(7-8), 381-390.

Minoci, I. \& Bregović, A., 2008: Grbice (Lepidoptera, Geometroidea, Geometridae) zbirke Košćec, in: Franjo Košćec i njegovo djelo 1882.-1968.: zbornik radova sa znanstvenog skupa. Presented at the Franjo Košćec i njegovo djelo 1882.-1968., Varaždin, pp. 129-168.

MıносI, I., 2012: Raznolikost grbica (Lepidoptera, Geometridae) Hrvatske i ekološka uvjetovanost njihove visinske rasprostranjenosti (PhD). University of Zagreb, Faculty of Science, Zagreb.

Mladinov, L., 1958: Popis noćnih leptira (Noctua) Zagreba i okolice. Hrvatski narodni zoološki muzej. Zagreb, 61 pp.

Mladinov, L., 1976: Lepidoptera iz doline gornjeg toka rijeke Kupe. II. Bombyces i Sphings. Acta entomologica Jugoslavica. 12(1-2), 89-98.

NowAckI, J., 1998: The noctuids (Lepidoptera, Noctuidae) of Central Europe. František Slamka, Bratislava, $51 \mathrm{pp}$.

Schmidt, B.C. \& Roland, J., 2009: Moth Diversity in a Fragmented Habitat: Importance of Functional Groups and Landscape Scale in the Boreal Forest. Annals of the Entomological Society of America 99, 1110-1120.

Slamka, F., 2006: Pyraloidea of Europe (Lepidoptera) 1. Pyralinae, Galleriinae, Epipaschiinae, Cathariinae \& Odontiinae. František Slamka, Bratislava, 138 pp.

Slamka, F., 2008: Pyraloidea of Europe (Lepidoptera) 2. Crambinae \& Schoenobiinae. František Slamka, Bratislava, 223 pp.

Slamka, F., 2013: Pyraloidea of Europe (Lepidoptera), Volume 3. Pyraustinae \& Spilomelinae. František Slamka, Bratislava, 357 pp.

Vignjević, G., Zahirović, Ž., Turić, N. \& Merdić, E., 2010: Moths (Lepidoptera: Heterocera) of Kopački rit Nature Park - Results of preliminary research. Entomologia Croatica 14, 17-32.

Vukotinović, L., 1879: Fauna leptirah u okolišu Zagrebačkom. Jugoslavenska akademija znanosti i umjetnosti, Zagreb, 129 pp. 


\section{SAŽETAK}

\section{Fauna noćnih leptira (Lepidoptera: Heterocera) Parka prirode Medvednica}

T. Koren

Na području Hrvatske nalazi se 11 Parkova prirode raspoređenih u sve tri biogeografske regije. Fauna noćnih leptira većina Parkova prirode nije bila sustavno istraživana, s iznimkom Kopačkoga Rita gdje su do sada izvršena preliminarna istraživanja noćnih leptira. Noćni su leptiri važan dio ekosustava, a s preko 160,000 opisanih vrsta važni su oprašivači mnogih biljnih vrsta. Znanje o raznolikosti noćnih leptira nekoga područja može olakšati upravljanje tim područjem te usmjeriti konzervacijske akcije. Park prirode Medvednica nalazi se u neposrednoj blizini Grada Zagreba, no fauna noćnih leptira toga područja nikada nije bila sustavno istraživana. Pregledom literature potvrđena je prisutnost tek 69 vrsta zabilježenih unutar granica Parka prirode. Recentnim istraživanjem provedenim između 2015. i 2019. godine na području Parka prirode Medvednica zabilježeno je 437 vrsta noćnih leptira, od kojih je njih 388 prvi put zabilježeno na tom području. Sveukupno je od sada na području Parka prirode Medvednica zabilježeno 455 vrsta noćnih leptira. Jedna vrsta porodice Tortricidae, Phtheochroa annae, po prvi puta je zabilježena u fauni Hrvatske. Ipak, popis noćnih leptira nije potpun te sadrži tek oko $40-50 \%$ potencijalne faune tog područja; no nakon ovog istraživanja područje Parka prirode Medvednica može se smatrati jednim od najbolje istraženih područja u Hrvatskoj. Na većini posjećenih područja diljem Medvednice stanišni uvjeti povoljni su za kratkoročni opstanak većine vrsta, ali zarastanje livada i rubova šume, kao i intenzivna košnja vršnih livada, mogli bi u u bliskoj budućnosti dramatično pogoršati održavanje velike biološke raznolikosti područja. Osim toga je važno održati i poboljšati komunikaciju leptira između izoliranih livadnih staništa diljem Medvednice održavanjem i stvaranjem šumskih rubova bogatih podrastom. 